\title{
Imaging the pancreas: from ex vivo to non-invasive technology
}

\author{
D. Holmberg • U. Ahlgren
}

Received: 28 May 2008 / Accepted: 5 August 2008 /Published online: 6 September 2008

(C) Springer-Verlag 2008

\begin{abstract}
While many recently published reviews have covered non-invasive nuclear imaging techniques, the aim of this review is to focus on current developments in optical imaging technologies for investigating the pancreas. Several of these modalities are being developed into non-invasive, real-time monitoring routines for pancreatic diseases. However, they also provide pre-clinical ex vivo and/or intravital tools for three-dimensional quantitative assessments of cellular and molecular events, with levels of specificity and resolution difficult to achieve with other currently available modalities.
\end{abstract}

Keywords Beta cell mass · Diabetes · Imaging · Intravital · Invasive $\cdot$ Non-invasive $\cdot$ Optical $\cdot$ Tomography

$\begin{array}{ll}\text { Abbreviations } \\ \text { BLI } & \text { bioluminescent imaging } \\ \text { 3D } & \text { three-dimensional } \\ \text { MRI } & \text { magnetic resonance imaging } \\ \text { OCT } & \text { optical coherence tomography } \\ \text { OPT } & \text { optical projection tomography } \\ \text { PET } & \text { positron emission tomography } \\ \text { SPECT } & \text { single photon emission computed tomography }\end{array}$

\section{Introduction}

For pre-clinical and clinical assessments alike, technological limitations have hampered many areas of diabetes

D. Holmberg

Department of Medical Biosciences, Umeå University,

S-901 87 Umeå, Sweden

U. Ahlgren $(\varangle)$

Umeå Centre for Molecular Medicine, Umeå University,

S-901 87 Umeå, Sweden

e-mail: Ulf.Ahlgren@ucmm.umu.se research. The scattered organisation of the islets of Langerhans within the much larger exocrine parenchyma puts high demands on the tools used, and even in small animals such as rodents, attempts to calculate the entire pancreatic beta cell content have been described as a true stereological challenge [1]. Hence, it is generally accepted that technologies contributing to better quantitative and spatial analyses of the pancreas and its disease processes would greatly improve our ability to study important aspects of both type 1 and type 2 diabetes. During recent years, significant efforts have been made to overcome the technological hurdles associated with pancreas imaging. A major aspiration has been to develop a strategy for the non-invasive monitoring of dynamic processes in the pancreas, including changes in beta cell mass and the inflammatory process during the development of type 1 and type 2 diabetes. Such a technology would have tremendous clinical value, spanning from early prediction/diagnosis of type 1 diabetes to monitoring the response to potential beta cell restoration schemes.

In this review we will discuss recent advances in imaging technology with direct relevance for diabetes research. Since several excellent reviews have been published that focus on recent progress in the development of non-invasive pancreas imaging based on nuclear imaging techniques, such as magnetic resonance imaging (MRI) and positron emission tomography (PET) (see, for example [2, $3]$ ), the focus here will be on optical techniques. These include ex vivo modalities that are currently applied primarily to pre-clinical diabetes research but have the potential to expand our scientific understanding of the disease, and may prove clinically relevant over the longer term. Generally speaking, across the range of techniques described here, the spatial resolution of the approach is inversely correlated with imaging depth. At one end of the spectrum, non-optical whole animal imaging displays the lowest resolution; at the other end, genuine microscopy 
(confocal) has the highest imaging resolution but the lowest depth penetration.

\section{Towards non-invasive monitoring of the pancreas and its diseases}

Although outside of the scope of this review, it should be emphasised that progress towards non-invasive monitoring of pancreatic disease processes has been made in many of the existing non-optical imaging modalities, including MRI, PET and single photon emission computed tomography (SPECT), as well as for different optical modalities over recent years [2-5]. In spite of this, significant technological issues must be resolved before a set-up for routine monitoring of beta cell mass for use in humans becomes a reality. Among the most critical challenges are the need for increased sensitivity to allow minor changes in beta cell mass to be scored, and a means of identifying markers not affected by altered beta cell function. Some of the most promising progress in non-invasive imaging of the pancreas has been made based on the MRI platform. Various mechanisms for contrast enhancement and for increasing specificity for the pancreas and its disease processes have been reported. One example, performed in a mouse model of type 1 diabetes, is the use of monocrystalline iron oxide nanoparticles to follow the alterations in the microvasculature that accompany insulitis by MRI [6]. The MRI modality has been most successful, however, when applied to the non-invasive monitoring of transplanted islets [7-9]. For this purpose, it appears possible that technologies such as MRI and PET could be applied in clinical settings in the near future. Of note, PET facilitates the pre-surgical discrimination of focal vs diffuse lesions in children with congenital hyperinsulinism and therefore represents an important clinical instrument in this setting [10]. One of the main challenges for MRI and PET in terms of the non-invasive determination of beta cell mass is to identify tissue-/cell-specific tracers. Promising results have been obtained for PET imaging using vesicular monoamine transporter 2 (VMAT2) as a surrogate marker for insulin production ([11] and references therein). However, for the monitoring of transplanted islets, this issue can at least in part be circumvented by in vitro labelling of the structures to be monitored. The search for a clinically meaningful scoring system for the progression of insulitis and beta cell loss during diabetes has led to interesting advancements in several of the nuclear imaging modalities, including the development of more specific tracers and increased resolution, as well as the use of different combinations of these modalities. Efforts to provide this will involve the development and use of new and better intravital (i.e. surgically exposed) or ex vivo- based imaging platforms that allow high-resolution, 3D spatial and whole organ assessment of the molecular and cellular processes underlying pancreas pathology. Moreover, non-invasive imaging (e.g. of beta cell mass) may not by itself be sufficient to gain an understanding of the full complexity of the disease. Visualisation and/or quantification of other cell types or structures that contribute to or are affected by the disease will be of the utmost importance for the provision of more substantial mechanistic insights. Hence, there is clearly a niche for the development of imaging platforms with an enhanced ability of visualising specific molecular distributions within the pancreas. Such technological improvements will be imperative to further our understanding of the cellular and molecular dynamics of the aetiology of type 1 diabetes and to obtain information on beta cell mass, including spatial, temporal and quantitative data on different cellular subtypes involved in the autoimmune attack-information likely to be critical for the successful design of immunotherapies.

\section{Nuclear imaging techniques}

Principle of detection

- MRI - nuclear magnetic resonance; PET/SPECT tomographic detection of photons emitted as a result of decaying radioactive isotopes

Strengths

- Non-invasive, excellent imaging depth, resolution (MRI), sensitivity (PET/SPECT)

Weaknesses

- Resolution (PET/SPECT) and limited source of contrast agents

Recent/further developments

- New and better contrast agents, increased resolution

\section{Bioluminescence imaging}

Bioluminescence imaging (BLI) differs from the other approaches described in this review in that it could be more accurately described as an optical technique for quantification of beta cell mass rather than imaging. Bioluminescence refers to the enzymatic generation of visible light by living organisms. In experimental research, this most commonly translates to the use of transgenic reporters derived from the North American firefly (Photinus pyralis) gene encoding luciferase. The enzymatic catalysis of the luciferase substrate D-luciferin results in the emission of photons at wavelengths that can be detected by a photosensitive detector, normally a charge-coupled device 
camera. Hence, BLI systems detect a continuous current of photons, emitted from the site of expression of the gene encoding luciferase, by an optical sensor at the surface of the animal. As such, the technique has been used for successful imaging of biological processes, including vasculogenesis, apoptosis and inflammation, in a variety of tissues. From a diabetological perspective, BLI has primarily been used for the non-invasive monitoring of beta cell mass. Using reporter mice expressing the gene encoding luciferase under the control of the rat or mouse Ins 1 promoter, it has been demonstrated that islet mass in situ can be quantified as a function of the activity of the gene, under both normal and metabolically disturbed conditions [12, 13]. Furthermore, BLI of islets isolated from such animals or islets expressing the gene encoding luciferase following virus-mediated gene transfer provides a promising approach for the evaluation of interventions aimed at prolonging islet graft survival [14-16]. Beta cell mass aside, BLI has also been successfully used to analyse other processes associated with diabetes aetiology or therapeutic interventions. These include the study of aspects of cell trafficking during the insulitis process in animal models of type 1 diabetes [17] and the monitoring of the immune response in islet transplantation experiments [18]. BLI is very sensitive and allows the detection of as few as ten islets when transplanted under the renal capsule or in the liver [14]. Another benefit is the relative ease with which large-scale longitudinal analyses can be performed. However, as pointed out by several researchers, BLI is influenced by a number of parameters, and these have to be carefully characterised to ensure its accuracy for quantifying islet beta cell mass, whether transplanted or in situ. Importantly, the catalysis of D-luciferin requires several cofactors, and additional studies are required to establish a linear relationship between photon generation and islet mass under different physiological conditions. Further, the potential influence of the physical condition of and/or functional changes in the islets on the bioluminescent output must be carefully validated [15]. Two-dimensional (planar) BLI is not capable of resolving depth, and photon attenuation is non-linear with respect to tissue depth and optical heterogeneity. Therefore, parameters such as anatomical differences in overlaying structures, post-surgical effects (in grafting studies) and imaging angle may also hamper quantitative assessments [19]. Potential approaches to circumvent such impediments are under development. By combining the most recent systems for diffuse bioluminescence tomography (BLT) with information of tissue heterogeneity provided by other imaging modalities such as MRI, it should be possible to more accurately correct for obstructions of this kind. In summary, BLI holds great potential for experimental diabetes research, primarily as a small animal imaging modality.

\section{Bioluminescence imaging}

Principle of detection

- Optical detection of photons emitted from luciferasemediated catalysis of luciferin

Strengths

- Non-invasive quantitative analyses in experimental animals

Weaknesses

- Resolution and limited ability to resolve depth

Recent/further developments

- Combinatorial approaches to obtain better 3D information

\section{Confocal microscopy}

Established for routine laboratory use in the 1980s, numerous studies have exploited the superior resolution of confocal microscopy to study different aspects of pancreas biology. These have included analyses ranging from ex vivo assessments of individual islet physiology [20], to more recent approaches for obtaining cellular/ subcellular resolution 3D information on molecular components and signalling sources during pancreas development [21]. For many applications, two photon laser scanning microscopy (TPLSM) [22], operating in the infrared spectrum, has several advantages over confocal laser scanning microscopy (CLSM), including reduced background disturbances, lower phototoxicity and, importantly, deeper penetration into the tissue $(\sim 0.1-0.5 \mathrm{~mm})$. Despite the limited penetration depth, several examples of new applications for confocal microscopy in diabetology research are emerging. Notably, an approach for intravital confocal imaging of islets in the tail of the pancreas of anaesthetised mice was recently presented [23]. In combination with immunological approaches for the induction of type 1 diabetes, the method has potential for in vivo monitoring of key players during the autoimmune attack that transgenically express fluorescent proteins. Hence, the methodology could help address questions such as how long effector cells reside within an islet or how many are required to ablate the beta cell mass. Another approach for confocal microscopy imaging of islet biology in live mice takes advantage of the eye cornea as a natural body window [24]. Hereby, the vascularisation and beta cell function of islets engrafted to the mouse iris can be repetitively monitored under both normal and pathological conditions. These types of technological platforms represent important steps for the intravital imaging of islet physiology and indicate that, although inappropriate for obtaining overall information of the 
pancreatic state, confocal microscopy will constitute a valuable asset for furthering our understanding of the mechanistic aspects of diabetes aetiology for years to come.

\author{
Confocal microscopy \\ Principle of detection \\ - Optical sectioning \\ Strengths \\ - Very high resolution and availability of contrast agents \\ Weaknesses \\ - Imaging depth—not suitable for whole organ analyses \\ Recent/further developments \\ - New platforms for intravital imaging
}

\section{Optical projection tomography}

Perhaps the most recent imaging modality pertinent to diabetes research is optical projection tomography (OPT) ([25], reviewed in [26]). OPT was originally developed as an ex vivo technology for the 3D visualisation of embryonic-scale specimens, and as such has facilitated studies of pancreatic development [27, 28]. Despite being an optical imaging modality, the 3D images generated by OPT are large volumetric data sets similar to those generated by other tomographic techniques (as opposed to optical sectioning techniques such as confocal microscopy); therefore, the same visualisation techniques may be used. Hence, OPT could be described as a CT scanner which, instead of using X-rays, employs light in the visible part of the spectrum. By combined improvements in sample preparation, tomographic imaging and computational processing, the technology was recently adapted to image specifically labelled structures within intact adult mouse organs, including the pancreas [29]. By the nature of the tomographic process, this allows for whole organ assessments of islet beta cell volume and distribution. In this respect, for ex vivo samples, OPT provides a unique quantitative resolution for the calculation of, for example, beta cell mass, by providing volumetric data and spatial coordinates of individual islet beta cell volumes, even when applied to intact specimens of pancreas [29] (Fig. 1). Detecting both transmitted and emitted light, OPT also has the benefit of permitting visualisation of structures labelled with standard laboratory markers such as antibodies specific for the protein of interest. OPT thus offers a clear advantage over other imaging modalities capable of whole pancreas imaging, in that the abundant pool of available antibody markers pertinent to diabetes research may be utilised as contrast agents. Currently available commercial OPT scanners allow for detection of two specifically antibody-tagged cell signals plus a general anatomy channel (autofluorescence) that provides information about the pancreatic parenchyma. Hence, in contrast to imaging modalities that lack the capacity for simultaneous detection, OPT appears ideally suited for monitoring cellular interactions and ratios on the whole organ scale. Attempts to further increase this capacity for simultaneous detection, by means of multispectral imaging, have been initiated (J. Sharpe, Centre for Genomic Regulation, Barcelona, Spain, personal communication). As such, the OPT technique is likely to play an important role in high-resolution ex vivo assessments aimed at addressing the overall state of the pancreatic constitution in rodent models of diabetes. By virtue of its medium/high throughput capacity, this may include analyses regarding the spatio-temporal dynamics of beta cell degradation/lymphocyte infiltration in the aetiology of type 1 diabetes, the evaluation of beta cell restoration schemes or screening for

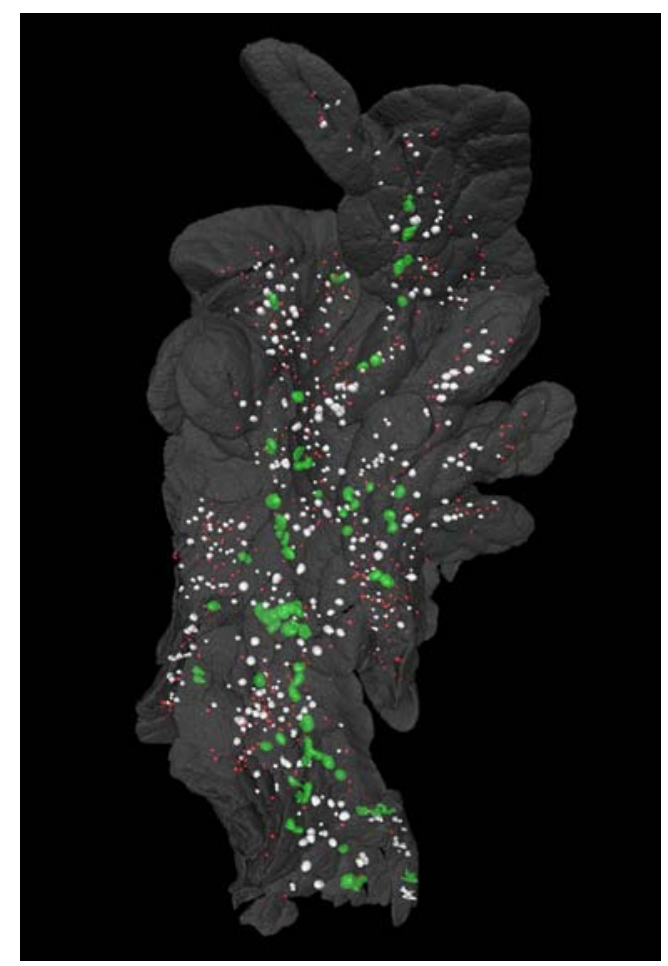

Fig. 1 OPT-based iso-surface reconstruction of pancreas from a NOD mouse (splenic lobe at 8 weeks). The pancreas outline is based on the background fluorescence signal (grey) whereas the insulin-producing cells of the islets (red, green and white) are based on the signal from insulin-specific antibodies. The technique allows for extraction of individual islet beta cell volumes, islet number and islet coordinates throughout the mouse pancreas. In the example shown, the different colours represent different size intervals. Red, $(6.9-200) \times 10^{3} \mu^{3}$; white, $(200-3,000) \times 10^{3} \mu \mathrm{m}^{3}$; green: $>3,000 \times 10^{3}{\mu \mathrm{m}^{3}}^{3}$. The size of the specimen is $\sim 1.4 \times 0.7 \mathrm{~cm}$ 
rare events or cell niches. Furthermore, it may represent a useful verification tool in the development of non-invasive strategies for islet mass monitoring by providing a straightforward protocol for post vivo corroborations of contrast agent uptake efficiency and/or the quantitative read-out from such technologies. At present, the mouse pancreas (or pancreatic biopsy specimens of a corresponding size, $\sim 1 \mathrm{~cm}$ in diameter) represents the upper size limit for the imaging of a specimen with OPT. The spatial resolution of whole organ assessments of such specimens is nearly cellular $(\sim 15-$ $20 \mu \mathrm{m})$. However, given the relative youth of the OPT technology, further improvements in these parameters are likely to emerge in the near future. Because of the optic properties of living tissue, OPT is not likely to become a tool for non-invasive assessments of the pancreas. However, the technique was recently adapted for four-dimensional imaging of organ cultures [30]. This technique may possibly be applied to pancreatic specimens, at least for studies of a developmental nature.

\section{Optical projection tomography \\ Principle of detection \\ - Optical tomographic detection of emitted or transmitted light \\ Strengths \\ - Resolution, available contrast agents and quantitative accuracy \\ Weaknesses \\ - Does not allow for non-invasive assessments \\ Recent/further developments \\ - Multispectral capacity and increased resolution}

\section{Optical coherence tomography}

Optical coherence tomography (OCT) represents an exciting technology with potential for monitoring islet distributions in the pancreas. This imaging modality is often described as the optical equivalent of ultrasound imaging, but instead of sound waves, photons are sent into the specimen, and those reflected by the tissue are detected [31]. This type of interferometric detection allows imaging relatively deep into the tissue and records intrinsic contrast without the need for labelling or sectioning of the sample. Depending on the optical scattering properties of the specimen, imaging depths of several hundred micrometres can be achieved, with high sensitivity and axial resolution. OCT imaging has emerged as a potent diagnostic tool in a variety of clinical settings. Endoscopic OCT has been employed to examine the histology of the gastrointestinal tract lining, e.g. in coeliac disease [32], and as a tool for assessing epithelial lesions of the main pancreatic duct [33]. One potential drawback of OCT is that it is unable to detect fluorescence and this limits its possibilities for detecting gene expression or protein production. Nevertheless, preliminary observations show that islets backscatter light differently from neighbouring pancreatic tissue, leading to clear images of small and large islets within fixed or unfixed tissue, in vitro or in intravital preparations. In the latter setting, the application of an extended focus scheme [34] allowed illumination over an axial range of more than $300 \mu \mathrm{m}$, with a lateral resolution of $2 \mu \mathrm{m}$ in live mice (Villiger, Goulley, Leitgeb, Lasser, Grapin-Botton, Meda, personal communication). Although the ability of OCT to quantify islet mass remains to be determined, it may become a useful tool for longitudinal studies of islet distribution/mass in experimental animals, provided improvements are made in terms of penetration depths. Furthermore, similar to OPT, the technique may assist in the verification of probe development for other noninvasive modalities.

\section{Optical coherence tomography}

Principle of detection

- Backscattering of near infrared light

Strengths

- Resolution and the potential to image islets without the addition of contrast agents

Weaknesses

- Imaging depth

Recent/further developments

- Increased imaging depth, longitudinal islet mass

monitoring

\section{Concluding remarks}

The development of imaging modalities that allow the detailed monitoring of cellular and molecular events in pancreas physiology and during disease development are much warranted and are definitely under way. While the 'holy grail' of this quest remains the establishment of a clinically applicable non-invasive technique with sufficient specificity and resolution to allow monitoring of, for example, the sequential loss of beta cell mass and the progressive insulitis during diabetes or the reversal of this process during therapeutic regimens, other important goals are at stake. An example of this is the need to dissect the early events in the 


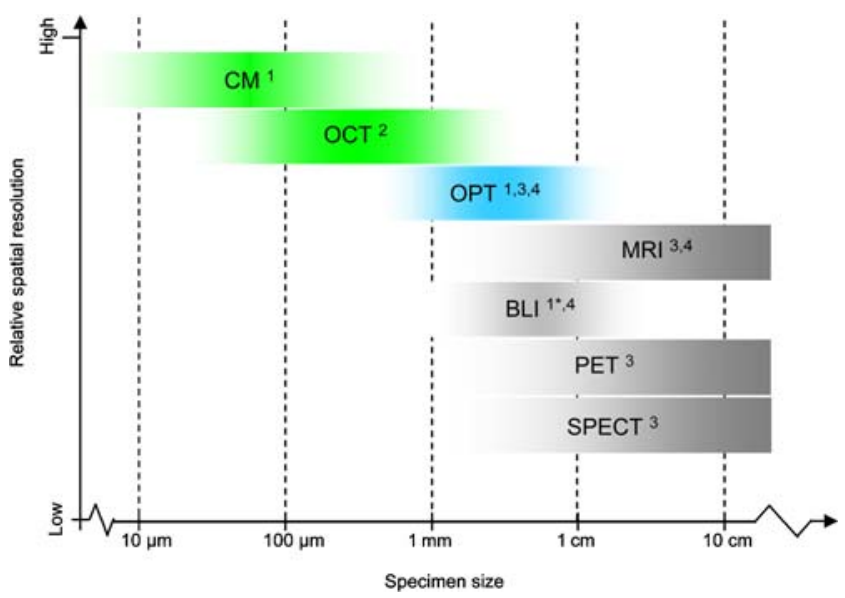

Fig. 2 Relative spatial resolution, at common specimen sizes, obtained by different imaging modalities when used to image the pancreas and its substructures. Note that the representation does not aim to describe the theoretical limit for each parameter; rather, it is intended as a guide to how the described techniques are currently used and the resultant output. Obviously, there is a significant overlap between several of the techniques in this respect. In addition, the spatial resolution of each approach is inversely correlated with imaging depth, and thus a more correct representation would describe the spatial resolution as a function of imaging range as diagonal lines rather than horizontal bars. The current representation has been selected for simplicity. Figures in superscript indicate that the technique allows for/merits from: ${ }^{1}$ an abundance of available contrast agents; ${ }^{2}$ detection of islets without using contrast agents; ${ }^{3}$ whole organ imaging; ${ }^{4}$ whole organ quantification. ${ }^{*}$ By means of transgenically expressed reporters. CM, confocal microscopy

pathogenesis of type 1 diabetes through the molecular analysis of cell-cell and cell-extracellular matrix interactions underlying extravasation, islet invasion and beta cell destruction mediated by the different subsets of leucocytes known to take part in this process. As outlined in Fig. 2, these analyses will require a combination of complementary modalities of molecular imaging that are informative at multiple levels and will include approaches that may dissect events at the levels of the organ and tissue, as well as at the cellular and subcellular levels. The cellular and molecular analysis of the progressive events underlying beta cell dysfunction in type 2 diabetes and the fate of explanted islets in diabetes therapy will require a similar approach.

While imaging modalities such as MRI and PET presently represent the methodologies of choice for noninvasive imaging of disease processes for the mouse and/or human pancreas, a technology such as OPT would appear to be more suited for monitoring 3D spatial relationships and quantitative processes across the whole organ, as required for the above type of applications. At present, high-resolution modalities such as confocal microscopy are required as a complement to, for example, OPT, to provide the means for cellular and subcellular dissection of these processes.
Other combinations of imaging modalities will provide the necessary tools for the further advancements of clinically applicable non-invasive techniques. Thus, ex vivo or intravital based technology platforms allowing whole organ 3D spatial analysis with high resolution and with specificity for molecular targets appear to be essential to drive this development. Further improvements in all of these techniques will be warranted and deserve continued efforts.

Acknowledgements The authors are grateful to J. Sharpe (Centre for Genomic Regulation, Barcelona, Spain), A. Grapin-Botton (Polytechnic School of Lausanne, Switzerland), H. Edlund (Umeå Centre for Molecular Medicine, Umeå University, Umeå, Sweden) for helpful comments on the manuscript. At the latter institution, T. Alanentalo is acknowledged for help with figures and $\mathrm{K}$. Loffler for editorial comments.

Duality of interest The authors declare that there is no duality of interest associated with this manuscript.

\section{References}

1. Skau M, Pakkenberg B, Buschard K, Bock T (2001) Linear correlation between the total islet mass and the volume-weighted mean islet volume. Diabetes 50:1763-1770

2. Souza F, Freeby M, Hultman K et al (2006) Current progress in non-invasive imaging of beta cell mass of the endocrine pancreas. Curr Med Chem 13:2761-2773

3. Virostko J, Jansen ED, Powers AC (2006) Current status of imaging pancreatic islets. Curr Dia Rep 6:328-332

4. Eich T, Eriksson O, Sundin A et al (2007) Positron emission tomography: a real-time tool to quantify early islet engraftment in a preclinical large animal model. Transplantation 84:893-898

5. Paty BW, Bonner-Weir S, Laughlin MR, McEwan AJ, Shapiro AM (2004) Toward development of imaging modalities for islets after transplantation: insights from the National Institutes of Health Workshop on Beta Cell Imaging. Transplantation 77:1133-1137

6. Turvey SE, Swart E, Denis MC et al (2005) Noninvasive imaging of pancreatic inflammation and its reversal in type 1 diabetes. $\mathrm{J}$ Clin Invest 115:2454-2461

7. Evgenov NV, Medarova Z, Dai G, Bonner-Weir S, Moore A (2006) In vivo imaging of islet transplantation. Nat Med 12:144-148

8. Jirak D, Kriz J, Herynek V et al (2004) MRI of transplanted pancreatic islets. Magn Reson Med 52:1228-1233

9. Kriz J, Jirak D, White D, Foster P (2008) Magnetic resonance imaging of pancreatic islets transplanted into the right liver lobes of diabetic mice. Transplant Proc 40:444-448

10. Hardy OT, Hernandez-Pampaloni M, Saffer JR et al (2007) Accuracy of $\left[{ }^{18} \mathrm{~F}\right]$ fluorodopa positron emission tomography for diagnosing and localizing focal congenital hyperinsulinism. J Clin Endocrinol Metab 92:4706-4711

11. Harris PE, Ferrara C, Baraba P, Polito T, Freeby M, Maffei A (2008) VMAT2 gene expression and function as it applies to imaging of $\beta$-cell mass. J Mol Med 86:5-16

12. Park SY, Wang X, Chen $Z$ et al (2005) Optical imaging of pancreatic beta cells in living mice expressing a mouse insulin I promoter-firefly luciferase transgene. Genesis 43:80-86

13. Smith SJ, Zhang H, Clermont AO et al (2006) In vivo monitoring of pancreatic beta-cells in a transgenic mouse model. Mol Imaging 5:65-75 
14. Chen X, Zhang X, Larson CS, Baker MS, Kaufman DB (2006) In vivo bioluminescence imaging of transplanted islets and early detection of graft rejection. Transplantation 81:1421-1427

15. Fowler M, Virostko J, Chen Z et al (2005) Assessment of pancreatic islet mass after islet transplantation using in vivo bioluminescence imaging. Transplantation 79:768-776

16. Lu Y, Dang H, Middleton B et al (2004) Bioluminescent monitoring of islet graft survival after transplantation. Mol Ther 9:428-435

17. Lee MH, Lee WH, Van Y, Contag CH, Liu CP (2007) Imageguided analyses reveal that non-CD4 splenocytes contribute to $\mathrm{CD}^{+}{ }^{+} \mathrm{T}$ cell-mediated inflammation leading to islet destruction by altering their local function and not systemic trafficking patterns. Mol Imaging 6:369-383

18. Roth DJ, Jansen ED, Powers AC, Wang TG (2006) A novel method of monitoring response to islet transplantation: bioluminescent imaging of an NF- $\mathrm{KB}$ transgenic mouse model. Transplantation 81:1185-1190

19. Virostko J, Chen Z, Fowler M, Poffenberger G, Powers AC, Jansen ED (2004) Factors influencing quantification of in vivo bioluminescence imaging: application to assessment of pancreatic islet transplants. Mol Imaging 3:333-342

20. Brelje TC, Scharp DW, Sorenson RL (1989) Three-dimensional imaging of intact isolated islets of Langerhans with confocal microscopy. Diabetes 38:808-814

21. Jorgensen MC, Ahnfelt-Ronne J, Hald J, Madsen OD, Serup P, Hecksher-Sorensen J (2007) An illustrated review of early pancreas development in the mouse. Endocr Rev 28:685-705

22. Denk W, Strickler JH, Webb WW (1990) Two-photon laser scanning fluorescence microscopy. Science 248:73-76

23. Martinic MM, von Herrath MG (2008) Real-time imaging of the pancreas during development of diabetes. Immunol Rev 221:200-213
24. Speier S, Nyqvist D, Cabrera O et al (2008) Noninvasive in vivo imaging of pancreatic islet cell biology. Nat Med 14:574-578

25. Sharpe J, Ahlgren U, Perry P et al (2002) Optical projection tomography as a tool for 3D microscopy and gene expression studies. Science 296:541-545

26. Sharpe J (2004) Optical projection tomography. Annu Rev Biomed Eng 6:209-228

27. Asayesh A, Sharpe J, Watson RP et al (2006) Spleen versus pancreas: strict control of organ interrelationship revealed by analyses of BapxI ${ }^{-/-}$mice. Genes Dev 20:2208-2213

28. Hecksher-Sorensen J, Watson RP, Lettice LA et al (2004) The splanchnic mesodermal plate directs spleen and pancreatic laterality, and is regulated by Bapx1/Nkx3.2. Development $131: 4665-4675$

29. Alanentalo T, Asayesh A, Morrison H et al (2007) Tomographic molecular imaging and 3D quantification within adult mouse organs. Nat Methods 4:31-33

30. Boot M, Westerberg H, Sanz-Esquerro J et al (2008) In vitro whole-organ imaging: 4D quantification of growing mouse limb buds. Nat Methods 5:609-612

31. Huang D, Swanson EA, Lin CP et al (1991) Optical coherence tomography. Science 254:1178-1181

32. Masci E, Mangiavillano B, Albarello L, Mariani A, Doglioni C, Testoni PA (2007) Pilot study on the correlation of optical coherence tomography with histology in celiac disease and normal subjects. J Gastroenterol Hepatol 22:2256-2260

33. Testoni PA, Mariani A, Mangiavillano B, Arcidiacono PG, Di Pietro S, Masci E (2007) Intraductal optical coherence tomography for investigating main pancreatic duct strictures. Am J Gastroenterol 102:269-274

34. Leitgeb RA, Villiger M, Bachmann AH, Steinmann L, Lasser T (2006) Extended focus depth for Fourier domain optical coherence microscopy. Opt Lett 31:2450-2452 